\title{
BMJ Open Exploring the causes and impacts of falls among ambulators with spinal cord injury using photovoice: a mixed- methods study
}

\author{
Hardeep Singh (D) , ${ }^{1,2}$ Arielle Shibi Rosen, ${ }^{2,3}$ Geoff Bostick, ${ }^{4}$ Anita Kaiser, ${ }^{1,2}$ \\ Kristin E Musselman ${ }^{1,2,5}$
}

To cite: Singh H, Shibi Rosen A, Bostick G, et al. Exploring the causes and impacts of falls among ambulators with spinal cord injury using photovoice: a mixedmethods study. BMJ Open 2020;10:e039763. doi:10.1136/ bmjopen-2020-039763

- Prepublication history and additional material for this paper are available online. To view these files, please visit the journal online (http://dx.doi. org/10.1136/bmjopen-2020039763).

Received 30 April 2020 Revised 11 July 2020 Accepted 23 July 2020

Check for updates

(C) Author(s) (or their employer(s)) 2020. Re-use permitted under CC BY-NC. No commercial re-use. See rights and permissions. Published by BMJ.

For numbered affiliations see end of article.

Correspondence to Dr Kristin E Musselman; Kristin.Musselman@uhn.ca

\section{ABSTRACT}

Objectives This study explored: (1) fall circumstances experienced by ambulators with spinal cord injury (SCl) over a 6-month period, (2) the impacts of fallsrelated injuries and fall risk and (3) their preferences/ recommendations for fall prevention.

Design A sequential explanatory mixed-methods design with two phases.

Setting A Canadian SCI rehabilitation hospital and community setting.

Participants Thirty-three ambulators with SCl participated in phase 1 and eight participants that fell in phase 1 participated in phase 2 .

Methods In phase 1, fall circumstances were tracked using a survey that was completed each time a participant fell during the 6-month tracking period. Phase 2 involved photovoice; participants took photographs of factors that influenced their fall risk and how their fall risk impacted their work/recreational activities. Participants discussed the photographs and topics related to fall prevention in an individual interview and a focus group.

Results of the 33 participants, 21 fell in 6 months.

Falls commonly occurred in the home while participants were changing positions or walking. Most falls occurred in the morning or afternoon. In phase 2, interviews and focus group discussion revealed three themes: (1) falls are caused by bodily impairments (eg, impaired reactive response during slips and trips and weakness and altered sensation in legs/feet), (2) impacts of fall-related injuries and fall risk (eg, psychosocial effects of fall-related injuries, limiting community participation due to the risk of falling and activity-dependent concern of falling) and (3) approaches to fall prevention (eg, fall prevention strategies used, components of fall prevention and utility of professional fall prevention strategies/interventions). Conclusions Fall prevention interventions/strategies should focus on minimising a person's fall risk within their home as most falls occurred in the home environment. Ambulators with $\mathrm{SCl}$ would benefit from education and awareness about common fall circumstances that they may encounter in their daily lives.

\section{INTRODUCTION}

Falls are a common and potentially detrimental complication of spinal cord injury
Strengths and limitations of this study

- A mixed-methods design allowed us to combine the strengths of qualitative and quantitative methods to generate detailed insights into factors related to falls and fall circumstances in ambulators with spinal cord injury.

- Photovoice allowed participants to be involved in the collection, analysis and development of actionoriented solutions.

- Falls were only tracked for a 6-month period, which limited quantitative analyses of season-related factors that influence falls.

- The fall survey did not inquire where in the home or community participants fell, and conclusions could not be drawn about specific rooms or locations.

- Different sample sizes and over-representation of older individuals in Phase 1 and 2 could impact the generalisability of the results/interpretations.

(SCI). Each year, $78 \%$ (95\% CI $73 \%$ to $83 \%$ ) of community-dwelling ambulators with SCI experience at least one fall. ${ }^{1}$ Living with a high risk of falling can lead to psychological stress, ${ }^{2}$ and up to $62 \%$ of ambulators with SCI will experience a fall-related physical injury. ${ }^{1}$ This can limit a person's participation in selfcare and productive activities, as well as his or her interactions with others. ${ }^{1}$ Considering these negative consequences of falls for individuals with SCI, it is necessary to develop effective strategies to reduce their frequency.

Maintaining ambulatory function within home and community environments is an important goal for many people with SCI. ${ }^{3}$ However, falls most commonly occur during standing or walking. ${ }^{4} 5$ Physical factors, including decreased or altered sensation, decreased strength in trunk and legs, and a loss of balance, are common contributors to falls. ${ }^{16}$ Additional contributors include having poor self-perceived balance and a fear 
of falling, as well as abusing prescription medication and alcohol. $^{6-9}$

While interest in fall prevention after SCI is growing, there is limited research related to ambulators with SCI, especially compared with research for those with other neurological conditions such as stroke, Parkinson's disease or multiple sclerosis. ${ }^{1}$ Recently, some insight was gained into the fall prevention strategies used by individuals with incomplete SCI through qualitative research. For example, slowing down and being cautious, being aware of the environment, modifying movement patterns, using assistive devices and avoiding risky situations were all described as strategies used by individuals with SCI in their daily lives to prevent falls. ${ }^{7}$ Another qualitative study $(\mathrm{n}=15)$ identified similar fall prevention strategies, including: exercising caution in or avoiding risky situations, screening the walking surface constantly, avoiding alcohol, using aids and moving slowly. ${ }^{2}$ However, prior qualitative studies have not comprehensively explored perceptions of participants concerning the reasons for falls. Also, prior quantitative studies have not explored in detail the nature and consequences of falls among this population. ${ }^{1}$ In order to design effective fall prevention solutions, there is a need to understand, through qualitative methods, the quantitative data that summarises the circumstances and causes of falls (ie, a mixed-methods approach must be employed).

An individual's fall prevention beliefs can influence their willingness to engage in specific prevention interventions. Therefore, the perspectives of the target population should inform the development of acceptable and feasible fall prevention solutions. ${ }^{10} \mathrm{~A}$ mixed-methods approach that combines qualitative and quantitative methods can generate a comprehensive dataset to further knowledge on falls in ambulators, and to express the fall prevention needs of the population. In this study, a mixed-methods approach was used to describe: (1) fall circumstances experienced over a 6 -month period, (2) the perspectives of ambulators with SCI on the impacts of falls on their lives and (3) the perspectives of ambulators with SCI on recommendations for fall prevention.

\section{METHODS}

\section{Design}

The study employed a sequential explanatory mixedmethods design with two phases. Phase 1 involved participants completing quantitative surveys to document fall circumstances over a period of 6 months. Phase 2 involved qualitative photovoice methodology to explore the quantitative data and generate new insights into the circumstances, consequences and prevention of falls. The 'Good Reporting of A Mixed Methods Study' guidelines were followed to increase the quality of reporting (see online supplementary material A). ${ }^{11}$

\section{Settings}

This study is part of a larger research project conducted at the Lyndhurst Centre, Toronto Rehabilitation Institute-University Health Network (UHN), which explores the causes and consequences of falls among individuals living with chronic SCI. All participants provided verbal and written informed consent to participate in this study.

\section{Patient and public involvement}

Patients or the public were not involved in the design, conduct, reporting or dissemination of our research.

\section{Participants}

Adults ( $\geq 18$ years of age) were purposefully recruited for phase 1 of this study if they met the following eligibility criteria: (1) had a chronic ( $\geq$ oneyear) SCI caused by a traumatic event; (2) had a neurological level of SCI between cervical (C) 1 to lumbar (L) 1 (American SCI Association Impairment Scale grade C-D); (3) identified walking as their main means of daily mobility and (4) were living in the community for $\geq 1$ month. Thirty-nine individuals expressed interest in the study and 33 were eligible and consented to participate in phase 1. Based on the recommended photovoice sample size of $10-12,{ }^{12} 14$ participants from phase 1 who had fallen were asked to participate in phase 2; eight participated in the individual interview, and five of these eight participated in the focus group discussion. The lead author had no established relationship/contact with participants prior to study commencement. During the screening, participants were informed of the study purpose and the lead researcher's occupation.

\section{Data collection}

Phase 1

Phase 1 involved tracking the frequency, circumstances and consequences of falls experienced by participants during a 6-month period. Falls were tracked using a previously developed survey. ${ }^{13}$ The survey contained 11 questions in total, with four open-ended responses allowing participants to provide additional details. Each participant completed an initial phone or in-person interview to provide demographic data and review the instructions for the survey. During the 6-month tracking period, participants were instructed to complete this online or paper-based survey within 24 hours of experiencing a fall. Participants were provided, verbally and in writing, with the definition of a fall as 'an event which results in a person coming to rest inadvertently on the ground or floor or other lower level. ${ }^{14}$ A monthly phone call was made to each participant to keep participants engaged in the study, remind them of the fall survey and to offer assistance with its completion. HS (a female PhD candidate and occupational therapist with qualitative research experience and interest in the research topic) collected the data for phase 1 . 


\section{Phase 2}

Phase 2 was a qualitative photovoice project. Photovoice was appropriate for this study since it positioned participants as the experts of the topic, and allowed them to be actively involved in the collection, analysis and development of action-oriented solutions. ${ }^{12}$ This phase involved three in-person or telephone meetings (researchers and participants) at the Lyndhurst Centre. During the first meeting (lasting $\sim 15 \mathrm{~min}$ ), participants were oriented to the method of photovoice including the privacy and ethical responsibilities. HS provided participants with verbal and written instructions for the photo assignment, which consisted of the following four questions that participants were asked to answer using photographs: (1) 'What increases your likelihood of falling', (2) 'What decreases your likelihood of falling', (3) 'How does the risk of falling affect your ability to participate in paid/ volunteer work' and (4) 'How does the risk of falling affect your ability to participate in recreational activities'. They were asked to capture at least two photographs for each question over 1 week. If participants had difficulty manipulating a camera, adaptations were available, or a caregiver was permitted to assist with photo taking.

The second meeting occurred within 1 week after completion of the photo assignment. It consisted of a semi structured interview (an hour long) with HS in which participants discussed the content depicted in their photographs. The SHOWeD framework, an acronym for the following five questions was used to facilitate photovoice interviews: "What do you see here? What is really happening here? How does this relate to our lives? Why does this problem, concern, or strength exist? What can we do about it?" 12 This framework was integrated into the interview guide to facilitate discussion.

The third meeting was a 2-hour long focus group facilitated by HS, ASR (female undergraduate student) and KEM (female Scientist and Assistant Professor). The goals were to discuss themes raised by the participants in the individual interviews and to brainstorm potential solutions or strategies to prevent falls. Each participant shared one of their photographs. The researchers then shared trends from the fall surveys and individual interviews with the participants. Participants were asked to reflect on and share their thoughts about the observed trends. Additionally, the participants were asked how information about falls after SCI could be shared to educate peers, healthcare professionals and the general public (see online supplementary material B for the interview guides). Field notes were made during and after the interviews and focus groups.

\section{Data analysis}

Phase 1

Demographic data were reported using descriptive statistics. Responses from the surveys were combined to calculate the incidence proportion of falls and fall-related injuries and the frequency of fall attributes (eg, time of day of fall). The incidence proportion was defined as the proportion of the sample that had a fall or fall-related injury during the tracking period. ${ }^{115}$

\section{Phase 2}

An inductive thematic analysis informed this study's qualitative data analysis. ${ }^{16}$ The semistructured interviews and focus group discussion were audio-recorded and transcribed verbatim by the lead author (HS). The transcribed dialogue was imported into NVivo V.12 (QSR International, Burlington, Massachusetts, USA) for management, coding and analysis of the data. HS and ASR independently coded the transcripts by reading each multiple times and summarised key segments into one or a few words. Preliminary themes were developed independently based on the relationships and patterns among these codes. HS and ASR then discussed their interpretations of the preliminary themes, and following this, KEM audited the qualitative analysis to verify that the identified themes closely reflected the viewpoints of the participants. After this audit, a set of final themes was developed.

\section{Trustworthiness}

The following methods were integrated into this study to support the trustworthiness of the results. ${ }^{1718}$ First, the key messages identified from the individual interviews were verified by participants during the focus group discussion, and this was used as a form of method checking. Second, multiple data collection methods were used to collect the data. This collection through varied modes allowed for method triangulation, which helped confirm the accuracy of the findings, and contributed to the generation of a rich understanding of the key themes. Finally, investigator triangulation was used, whereby multiple authors were involved in the data collection and analysis.

\section{RESULTS}

\section{Phase 1}

The demographics and injury characteristics of participants $(n=33)$ by falls status are displayed in table 1 .

\section{Incidence proportion of falls and fall-related injury}

A total of 87 falls were recorded (with a range of 1-15 falls per participant). Over the 6-month tracking period, $63.6 \%(n=21)$ of participants fell at least one time, and $71.4 \%(\mathrm{n}=15)$ of fallers experienced recurrent falls (ie, two or more falls).

Thirty-five fall-related injuries were reported by 15 participants. The most commonly reported injuries were bruises, pain and bumped heads. Detailed descriptions of the impacts of fall-related injuries and fall risk were identified in theme 2 .

\section{Fall attributes}

Most falls occurred inside the participant's home during the morning or afternoon (see table 2). Falls inside and just outside the home commonly occurred while the participants were changing positions (eg, 'bending down 
Table 1 Phase 1 participant demographics and injury characteristics

\begin{tabular}{|c|c|c|c|}
\hline Characteristics & $\begin{array}{l}\text { Total sample } \\
(n=33)\end{array}$ & $\begin{array}{l}\text { Fallers } \\
(n=21)\end{array}$ & $\begin{array}{l}\text { Non-fallers } \\
(n=12)\end{array}$ \\
\hline $\begin{array}{l}\text { Mean age (SD) } \\
\text { (years) }\end{array}$ & $61.6 \pm 12.6$ & $60.9 \pm 11.3$ & $62.8 \pm 15.1$ \\
\hline \multicolumn{4}{|l|}{ Biological sex (n) } \\
\hline Male & 23 & 16 & 7 \\
\hline Female & 10 & 5 & 5 \\
\hline $\begin{array}{l}\text { Mean (SD) time } \\
\text { post-SCI (years) }\end{array}$ & $11.3 \pm 12.1$ & $15.3 \pm 13.5$ & $4.3 \pm 3.7$ \\
\hline \multicolumn{4}{|c|}{ Neurological level of injury (n) } \\
\hline Cervical & 24 & 14 & 10 \\
\hline Thoracic & 7 & 5 & 2 \\
\hline Lumbar & 2 & 2 & 0 \\
\hline
\end{tabular}

$\mathrm{SCl}$, spinal cord injury.

to do up my coat', 'bending to pick up something', 'on hands and knees moving furniture' or 'adjusting concrete into place on patio') or walking. Two-thirds of falls in the community/workplace occurred while the participants were walking (see figure $1 \mathrm{eg}$, of fall risk during standing/ walking).

Almost half of the falls inside and just outside the home were caused by poor balance, legs giving out and weakness in the legs. The top factors contributing to falls experienced in the community and workplace were poor balance, tripping over something in the environment and slipping. In theme 1 below, participants provided detailed reflections on how their activities and the contributing factors caused falls in the home and community. ${ }^{19}$

\section{Phase 2}

Eight participants completed an individual interview. Five of these participants also participated in the focus group discussion; one participant was unavailable (ie, out of country) and two others agreed to participate via telephone, but were unreachable on the day of the focus group. Participant demographics and injury characteristics for those that completed phase 2 are displayed in table 3 .

The analysis of the qualitative data revealed three themes: (1) Falls are caused by bodily impairments, (2) Impacts of fall-related injuries and fall risk and (3) Approaches to fall prevention (see table 4 for a summary of the themes and subthemes).

\section{Theme 1: falls are caused by bodily impairments}

Theme 1 reflects discussion about fall trends from the surveys as well as other factors that contributed to participants' previous falls.

Subtheme 1a: impaired reactive response during slips and trips Surveys revealed that poor balance was a top perceived contributor to falls that occurred in the home and
Table 2 Fall circumstances

\section{Fall circumstance}

Falls ( 87 falls in total)

Time of fall $\left(\%, n^{\text {falls* }}\right)$

\begin{tabular}{lc} 
Morning & $35(40.2)$ \\
Afternoon & $28(32.2)$ \\
Evening & $16(18.4)$ \\
\hline Night & $8(9.2)$ \\
Location of fall $\left(\%, \mathrm{n}^{\text {falls } *)}\right.$ & \\
Home indoors & $43(49.4)$ \\
Community outdoors & $22(25.3)$ \\
Home outdoors & $11(12.6)$ \\
Community indoors & $8(9.2)$ \\
Workplace & $3(3.4)$
\end{tabular}

Activity by location

Inside or just outside the home $\left(\%, n^{\text {A.H*})}\right.$

$\begin{array}{lc}\text { Changing positions } & 19(35.2) \\ \text { Walking } & 16(29.6) \\ \text { Standing } & 5(9.3) \\ \text { Getting into/out of bed } & 4(7.4) \\ \text { Walking up or down the stairs } & 4(7.4) \\ \text { Getting into/out of a vehicle } & 2(3.7) \\ \text { Dressing } & 2(3 .) \\ \text { Other } & 1(1.9) \\ \text { Opening/closing a door } & 1(1.9)\end{array}$

Community or workplace $\left(\%, n^{\text {A.C*}}\right)$

Walking

$22(66.7)$

Standing

$4(12.1)$

Changing positions

$3(9.1)$

Getting into or out of a vehicle

$2(6.1)$

Sports/exercise

$1(3.0)$

Other

1 (3.0)

Causes of falls (could select $\geq 1$ for each fall) Inside or just outside the home $\left(\%, \mathrm{n}^{\mathrm{C} . \mathrm{H}_{*}}\right)$

Poor balance

$23(15.3)$

Legs gave out

$22(14.7)$

Weakness in legs

$21(14.0)$

Other $18(12.0)$

Doing more than one thing

$12(8.0)$

Tired $12(8.0)$

Tripped

$9(6.0)$

Slipped

8 (5.3)

Distracted

$7(4.7)$

Moving too quickly/rushing

$6(4.0)$

Dark/poorly lit environment

3 (2.0)

Dizzy

$3(2.0)$

Spasm in leg(s)

$3(2.0)$

Not using mobility aid

$1(0.7)$




\begin{tabular}{lc}
\hline Table 2 Continued & \\
\hline Fall circumstance & Falls (87 falls in total) \\
\hline Weather & $1(0.7)$ \\
\hline 'Don't know' & $1(0.7)$ \\
\hline Community or workplace $\left(\%, \mathrm{n}^{\text {C.C*})}\right.$ & \\
\hline Poor balance & $13(15.9)$ \\
\hline Tripped & $12(14.6)$ \\
\hline Slipped & $9(11.0)$ \\
\hline Legs gave out & $8(9.8)$ \\
\hline Other & $7(8.5)$ \\
\hline Moving too quickly/rushing & $7(8.5)$ \\
\hline Weather & $7(8.5)$ \\
\hline Tired & $5(6.1)$ \\
\hline Weakness in legs & $5(6.1)$ \\
\hline Doing more than one thing & $2(2.4)$ \\
\hline Dark/poorly lit environment & $2(2.4)$ \\
\hline Not using mobility aid & $2(2.4)$ \\
\hline Distracted & $2(2.4)$ \\
\hline Don't know & $1(1.2)$ \\
\hline Type of injury (\%, $\mathrm{n}^{\text {injuries*})}$ & \\
\hline Bruises & $19(40.4)$ \\
\hline Pain & $9(19.1)$ \\
\hline Cuts/scrapes & $8(17.0)$ \\
\hline Bumped head & $8(17.0)$ \\
\hline Other & $3(6.4)$ \\
\hline Medical attention required $\left(\%, \mathrm{n}^{\text {falls*})}\right.$ & \\
\hline No & $84(95.5)$ \\
\hline Yes & $4(4.5)$ \\
\hline
\end{tabular}

${ }^{*}$ Changing positions: 'Getting into and out of a body position and moving from one location to another, such as rolling from one side to the other, sitting, standing, getting up out of a chair to lie down on a bed, and getting into and out of positions of kneeling or squatting ${ }^{319}$; falls* denominator is total number of falls;

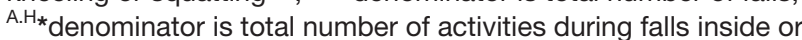
just outside the home; ${ }^{\text {A.C*}}$ denominator is total number of activities during falls that occurred in the indoor/outdoor community; ${ }^{C} . H_{*}$ denominator is total number of causes of falls that occurred inside or just outside the home; ${ }^{\text {C.C*}}{ }^{*}$ denominator is total number of causes of falls that occurred in the indoor/outdoor community;

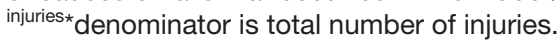

community. Several participants described impaired reactive responses when experiencing decreased balance, which increased their risk of falling. Participants explained that it was difficult for them to adjust the position of their feet to compensate for uneven walking surfaces, as altered sensation prevented reactive responses and adjustments. In addition, because some participants were unable to use their arms to maintain stability when unbalanced, they were more likely to fall. In her words, P5 compared her reactive responses to those of someone without SCI:
Your foot would adjust and comfortably slide or slip or partially fall, but you would be able to break your fall because your hands go in front of you. But unfortunately, I can't break a fall because my arms don't go out in front of me anymore to break a fall like they used to because of the injury...That's just an automatic thing when you're able-bodied...With me, I'm going to do a face plant because my arms just don't go out anymore since the injury.

\section{Subtheme $1 \mathrm{~b}$ : weakness and altered sensation in legs/feet}

Many participants spoke of altered gait patterns due to persistent weakness in their legs, and this increased their susceptibility to falls. Since some participants had difficulty dorsiflexing and plantarflexing their feet, they frequently tripped while walking over uneven surfaces. For instance, P2 described her unstable gait pattern as a tripping hazard: 'I should be going heel-toe, heel-toe, but sometimes it's more like shuffle-step-shuffle. And it's the shuffle that trips you.' Based on the surveys, falls due to 'tripping' were more common in the community compared with the home. Also, a few participants constantly dragged one or both of their feet while walking, and the others had a tendency to drag their feet when they were tired. This increased participants' likelihood of tripping.

When walking barefoot, the contact of the soles of participants' feet with objects frequently caused spasms. In addition, for many participants, stepping on or off curbs presented a high chance of falling due to weakness in their legs. For example, P1 explained that his knee would sometimes 'give out' when he stepped up onto a curb. The frequency of reported falls during standing and walking was corroborated by the survey responses.

\section{Subtheme 1c: altered body response caused by external factors}

Several participants explained that external factors caused altered and often unpredictable body responses, which increased their risk of falling. When the temperature was too hot or cold, some participants experienced altered sensation and/or uncontrollable movements in their legs. For example, P2 found that hot weather altered the sensation in her feet, explaining, 'Because of the heat [my feet] feel like they are made out of cement.' However, survey data revealed that 'weather' accounted for less than $4 \%$ of the total factors perceived to contribute to falls in the home and community. A few participants explained that when the weather was too cold, they experienced body sensitivity, spasms, and muscle stiffness that led to falls. P2 stated, 'When the temperature is cold my legs get really stiff and they just don't work properly. [My legs] give out on me.' P4 experienced chronic pain that became worse with specific environmental conditions. He explained that being outside on a cold day 'just for a couple of minutes' caused 'all these situations [to] arise that are unpredictable to how my body reacts'. On days that were humid, P6 compared walking to 'pushing your body through deep water...to propel your legs forward, 


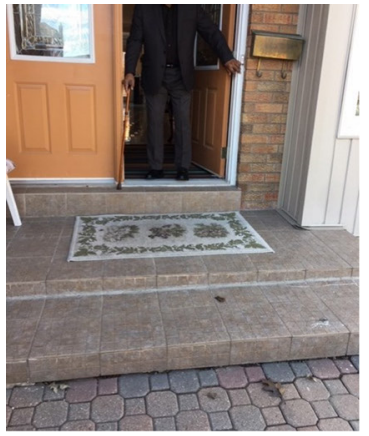

A: "Going out of the door, because there are so many levels to get down to the driveway... if you're using a walker, it's very unbalanced. I don't have the walker in that picture" (P4).

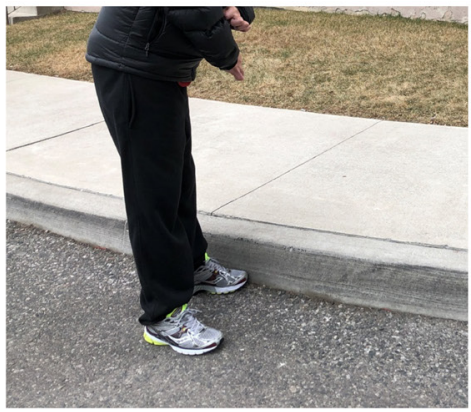

B: "For me to step up on that curb is too scary...A little curb - no problem...this action of transferring my weight...I can't trust this leg, that knee could give out" (P1)

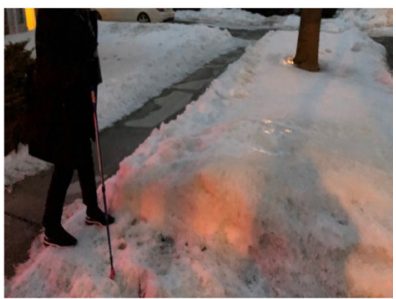

C: "Challenge of walking downhill in dark with ice and snow patches" (P5).

Figure 1 Factors that increase fall risk.

it's like you're walking in...water up to your chest.' These three factors (ie, 'weather', 'spasms' and 'legs giving out') accounted for nearly one-fifth of total contributors to falls cited by participants in the surveys.

For P1, altered body response was caused by external factors such as stress. P1 explained that his body's reaction to any form of stress resulted in an increased risk of falling: 'It's the central cord. My body wants to go into the fetal position. So any stress...let's say we were having this huge argument right, immediately you would see... my body wants to go into the fetal position.'

Alcohol was another factor that altered participants' perceived body responses and increased their risk of falling. Despite a higher risk of falling, one participant continued to consume alcohol to reduce his constant neuropathic pain. Although alcohol was discussed in the interviews as a common factor perceived to increase fall risk, it was not cited in the surveys as a common factor contributing to falls.

Table 3 Participant demographics and injury characteristics in phase 2

\begin{tabular}{lllll}
\hline Participant & & $\begin{array}{l}\text { 5-year } \\
\text { age } \\
\text { code }\end{array}$ & $\begin{array}{l}\text { Sex } \\
\text { category } \\
\text { time since } \\
\text { injury } \\
\text { category }\end{array}$ & $\begin{array}{l}\text { Neurological } \\
\text { level of } \\
\text { injury }\end{array}$ \\
\hline P1 $^{*}$ & Male & $50-54$ & $1-4$ & Cervical \\
\hline P2 $^{*}$ & Female & $55-59$ & $10-14$ & Cervical \\
P3 & Male & $50-54$ & $40-44$ & Thoracic \\
\hline P4 & Male & $65-69$ & $45-49$ & Thoracic \\
P5 & Female & $55-59$ & $10-14$ & Cervical \\
\hline P6 & Male & $45-49$ & $5-9$ & Cervical \\
P7* & Male & $50-54$ & $1-4$ & Cervical \\
\hline P8 & Male & $65-69$ & $20-24$ & Cervical \\
\hline
\end{tabular}

*Participated in the individual interview only.

Subtheme 1d: 'I was tired; that's why I was falling'

Fatigue accounted for $7.3 \%$ of the falls that were reported on the surveys. Participants explained that fatigue increased their chances of falls because it increased their likelihood to overlook environmental fall hazards. P2

Table 4 Themes and subthemes

Theme 1: Falls are caused by bodily impairments
Subtheme 1a: Impaired reactive response during slips and trips

Subtheme 1b: Weakness and altered sensation in legs/feet

Subtheme 1c: Altered body response caused by external factors

Subtheme 1d: 'I was tired; that's why I was falling'

Subtheme 1e: Pain affecting balance

Subtheme 1f: Optimal level of caution

Theme 2: Impacts of fall-related injuries and fall risk

Subtheme 2a: Psychosocial effects of fall-related injuries

Subtheme 2b: Limiting community participation due to the risk of falling

Subtheme 2c: Activity-dependent concern of falling

Subtheme 2d: 'I get to fall'

Theme 3: Approaches to Subtheme 3a: Fall prevention fall prevention strategies used

Subtheme 3b: Components of fall prevention

Subtheme 3c: Utility of professional fall prevention strategies/interventions

Subtheme 3d: Peer vs professional fall prevention training Subtheme 3e: Priorities for fall risk reduction and fall prevention 
recounted a period where she had several falls because she did not get enough rest. She stated that fatigue caused muscle weakness, which made her more susceptible to falling. Similarly, P8 explained that his cognitive awareness of the environment and reaction time were reduced when he was tired: 'Your balance is not just as physically adept when you are tired.'

\section{Subtheme 1e: pain affecting balance}

Many participants described the effect of pain, a common complication of SCI, on their gait patterns. Pain often reduced their balance and mobility, and for $\mathrm{P} 6$, pain in his legs caused him to 'start to limp more.' Also, pain in his upper extremity impacted his risk of falls as it reduced his ability to 'hold a cane.' When the pain was only experienced unilaterally, it caused participants to 'feel unbalanced' and increased their likelihood of falling.

\section{Subtheme 1f: optimal level of caution}

Participants believed that there was an optimal level of caution that ensured a lower risk of falling. Falls were perceived as more probable when participants were less cautious, since they were more likely to overlook specific fall risk factors. Participants explained that after having SCI, they had to direct more attention to their physical movements in order to prevent falls. Multitasking accounted for $6 \%$ of the falls reported on the surveys. If participants were preoccupied with one or multiple tasks and simultaneously walking or performing a transfer, they could no longer fully concentrate on their movements and were at a heightened risk for falls. Based on her experiences, P5 explained that she would likely fall if she did not direct her full attention to her movements: 'When my mind is off of [falls] and I'm not looking down, I'm gonna go down...my attention needs to be where my legs are, especially if there's uneven ground or incline, decline, pebbles, rock, ice.'

Distraction (representing 3.9\% of contributing factors reported on the surveys) interfered with a participant's ability to use visual and cognitive strategies to compensate for decreased balance and reduced sensation. For instance, P1 explained that when he was distracted and unable to use his vision to compensate for the decreased strength and sensation in his legs, his risk of tripping increased.

I have no real feeling in my left leg...if I walk down the road, I physically have to tell my brain to lift my leg... if I am walking and I get distracted...I am stumbling.

On the other hand, when participants were overly cautious, they were also at a higher risk of falling, but this factor was not captured on the surveys. Based on his experiences, $\mathrm{P} 4$ explained that anticipating a fall could trigger one: 'If I...walk normal, I tend to overcome [the fall risk]. But, if I'm focusing, 'Oh this place is treacherous, something's gonna happen' and that's when I notice a fall happens.' Thinking about a fall induced a physical response: 'because you're thinking that you're gonna fall, the body stiffens up' (P4). When P4's body stiffened, his balance and gait pattern were impacted, and he believed he was at a higher risk of falling.

\section{Theme 2: impacts of fall-related injuries and fall risk}

Participants described multiple effects of fall-related injuries and fall risk. The potential of falling and sustaining an injury led some ambulators with SCI to limit their community participation and develop an activity-dependent concern of falling. It is important to note that despite having had falls in the past, there were some participants who felt fortunate to be able to ambulate and to have the subsequent ability to fall.

\section{Subtheme 2a: psychosocial effects of fall-related injuries}

In addition to physical injuries, falls had psychosocial effects. As described by P3, the recovery from a physical injury could potentially limit the participants' ability to function for an extended period of time. P5 described a situation where she sustained an ankle sprain and visible cuts, scrapes and bruises on her face from a fall, and she avoided going into the community due to a feeling of embarrassment about the visible injuries. She explained, 'I was bruised you know my face was black and blue. It looked like someone punched me. It didn't look like I fell. I had an ankle sprain or foot sprain...I was kind of isolated in the house for a few weeks because makeup wouldn't cover it.'

\section{Subtheme $2 \mathrm{~b}$ : limiting community participation due to the risk of} falling

A few participants voluntarily limited their community participation to avoid situations that increased their risk of falling. Crowded places, including amusement parks, cultural dances, and other social events included an elevated risk for falls and were commonly avoided. P5 spoke about temporarily limiting her community participation during bad winter weather: 'I won't leave the house if it's a [snowy] day like that because of the risk of falling and the recovery from an injury is just so long.'

\section{Subtheme 2c: activity-dependent concern of falling}

Participants felt fearful of falling when they performed activities during which they had previously fallen. For example, P7 outlines the potential fear associated with environments where previous falls have occurred: 'These stairs, I fell once but I'm always concerned anytime I'm on the stairs. I'm scared.'

While living with a high risk of falling was universally stressful, the fear of falling did not limit all participants. For example, P5 experienced stress each time she was on an escalator but continued to go on escalators. She stated, 'Going down an escalator is a problem. First of all, because it's something moving...I walk slow. Also, if I get on, I could go fall forward. Even though I'm holding onto the handrail. It stresses the living life out of me.' 


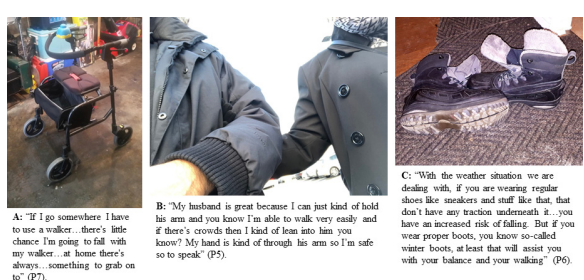

Figure 2 Strategies perceived to reduce fall risk.

\section{Subtheme 2d: 'I get to fall'}

Not all participants viewed falls in an entirely negative light. P1 explained that he was 'lucky' to regain walking function after his SCI, as he was not expected to walk again. He felt fortunate to have the ability to walk and he acknowledged that falls were an associated risk that he was willing to accept. Despite having had seven falls, three fall-related hospitalisations, and being placed on a concussion protocol in the last year, P1 explained: 'I get to fall...if I fall, I fall.' He elaborated: 'I feel that [falls are] part of my injury...I know there's a risk to participating in activities but [I] push through it.' This participant was willing to accept the risk of falling without allowing it to limit his participation in day-to-day activities.

\section{Theme 3: approaches to fall prevention}

This theme reflects the perspectives of participants on various aspects of fall prevention, including: (1) Fall prevention strategies used, (2) Components of fall prevention, (3) Utility of professional fall prevention strategies/ interventions, (4) Peer versus professional training for fall prevention and (5) Priorities for fall risk reduction and fall prevention.

\section{Subtheme 3a: fall prevention strategies used}

Figure 2 depicts images captured by participants of factors that reduced their fall risk.

\section{Scan the environment and be cognitively aware}

Participants outlined several strategies they employed to improve their balance when standing and walking. These strategies compensated for reduced sensation in their lower extremities, and largely involved visually scanning the environment and directing increased cognitive awareness to their gait pattern and floor transitions or unevenness in order to avoid tripping.

P8 made a conscious effort to walk with a 'wider stance' because it 'creates a little more stability.' P1 had to be constantly aware of his body's responses so that he could prepare to stretch his legs to stop them from giving out. When walking outside, P1 explained that he compensated for reduced sensation in his leg by consciously reminding himself to lift his leg while walking. Similarly, P8 shared his strategy to plan his movements in his mind to avoid potential fall risks where he had previously fallen. Many participants indicated that slowing down gave them an opportunity to plan movements, as well as anticipate and react to unexpected body responses. By slowing down and exhibiting increased levels of caution, participants were able to decrease their risk of falling.

\section{Getting to know one's environment}

Participants explained that familiarising themselves with their environments was an important strategy to prevent falls. P3 explained that falls were more prevalent during the first few years post-injury, but after he adjusted to his surroundings and living with SCI, he experienced fewer falls. 'In my case, I think in spaces of familiarity, like my home, I know it so well. I mean the first couple of years when I first moved there after my injury, it was about getting to know it.' Although this was considered a strategy to reduce fall risk, survey data revealed that most falls occurred in the home, an environment all participants were familiar with.

\section{Using or not using a mobility aid}

Although all participants agreed that a mobility aid could reduce their risk of falls, not all participants consistently used a mobility aid in their home or the community. In their home, several participants did not use a mobility aid (eg, walker) because it was not 'practical'; rather, they preferred to rely on objects in their environment for balance and support (figure 2A). Participants explained that relying on the environment for support was convenient and useful: 'Touching walls kind of helps me. If I lose balance, then I lean into the wall and the wall keeps me up' (P3). Other participants avoided mobility aids simply because they had limited space in their home.

Participants also described the situational possibility for a mobility aid to increase their fall risk. For instance, using a cane on a slippery surface presented a challenge for P6: 'The tip of the cane can even slip on the ice.' Other participants described instances in the community when they felt holding on to a caregiver or family member's arm for balance support was sufficient and they did not use their mobility aid (see figure 2B). In order to reduce falls, it was important to make an appropriate judgement call about when to use a mobility aid. For instance, P1 compensated for poor balance by using a power wheelchair within his home when he was under the influence of alcohol.

\section{Modified activities}

To prevent falls, participants often altered the way they participated in specific activities. For example, P3 explained that when his legs felt particularly weak, he would modify the way he went up a flight of stairs: 'almost like crawling up the stairs...going up in a crouched position with your hands.' Other participants spoke about modifying their participation in daily activities and sports/recreation to increase their safety and reduce the risk of falling.

\section{Footwear}

Appropriate footwear was important to reduce fall risks as shoes could compensate for reduced sensation and foot drop. Walking barefoot or in socks was generally 
understood to increase the risk of falling. For P5, it was necessary for a shoe to have a sole that had an upwards curved tip. She explained, 'Because of the foot drag, what I find with this shoe when you're walking...there's less catching...I'm wearing a running shoe...the toe curves upward, so when you're walking, it helps you create that natural like heel, ball, toe business.' For many, closed toe shoes reduced the risk of spasms caused by temperature sensitivity or wet floors. Thus, many participants wore shoes inside their homes, including in the shower. Winter boots with good grip were also a necessity in the winter months (see figure 2C).

\section{Asking for assistance}

Asking for assistance was identified as another strategy to reduce the risk of falling. However, asking for help from strangers could be difficult. P5 explained that taking someone (eg, a personal support worker (PSW), friend or family member) along to use for balance support on community outings was helpful, especially in a crowded place. Climbing steps with a gait aid was difficult for a few participants, and some only ascended or descended stairs while supervised by a caregiver. P1 found that when he gently touched someone's arm, it significantly increased his confidence in his balance.

\section{Getting up from a fall}

Participants used specific individual strategies to get up from a fall when they were alone in their homes. For instance, P1 recalled, 'I can remember specifically lying in the middle of my living room floor and panicking...I calmed down...I crawled over to the couch. I used my shoulder...I pushed my foot up on my TV stand and pushed back, and was able to get my back onto the couch...It took me an hour.'

\section{Technology to reduce fall risk}

Technological aids such as stair glides, automatic door openers, and power mobility aids were believed to reduce the risk of falls. For instance, a stair glide was used by those who perceived a high risk of falling on stairs, and an automatic door opener was suggested by a participant who had difficulty maintaining his balance while opening or closing a door.

\section{Subtheme 3b: components of fall prevention}

Participants recognised that a flexible programme would be necessary to accommodate everyone's needs, and participants considered education to be a vital part of fall prevention. They believed education should centre around recognising fall hazards in each person's home in order to provide individuals with the skills to reduce their daily fall risks. $\mathrm{P} 4$ explained that therapists should 'educate you about space in your home, especially because you will spend $90 \%$ of the time in your home, right?' A home safety assessment was believed to be a helpful way to recognise and mitigate the risk of falls within the home environment. P6 explained, 'If [a healthcare professional] could come home and help you sort out your surrounding...we are new to this, we don't see the danger.' A variety of formats were suggested to accommodate the diverse learning needs of individuals with SCI. For instance, P1 proposed 'Aside from giving the information in text, I think maybe if we give examples of scenarios in a video format'

\section{Subtheme 3c: utility of professional fall prevention strategies/} interventions

Participants had varying opinions regarding the value of professional fall prevention strategies and interventions. While several participants believed that fall prevention strategies/interventions would be helpful, others were sceptical that it could prevent falls or reduce their frequency. P1 believed that training could not possibly address all the potential risk factors since the causes of falls were complex and variable. He explained, 'Fall prevention, I don't think it would hurt. As far as helpful-I don't know, there's so many scenarios involved in any fall that anybody faces...You don't plan to fall...it happens so quick.' Similarly, P3 felt that fall prevention was an application of 'common sense,' and he believed that formal training was not needed. Conversely, P2 and P5 believed individuals with SCI should 'definitely' receive fall prevention education, as it would be helpful to reduce the frequency of falls.

\section{Subtheme 3d: peer versus professional fall prevention training}

Participants regarded healthcare professionals as the experts on fall prevention. P2 believed that therapists were particularly important shortly after SCI 'to get the guidance going in the right direction.' The fall prevention training provided by therapists could be reinforced by peer trainers, as indicated by some participants. However, some participants felt peers would not have sufficient training to discuss/advise ambulators about fall prevention.

\section{Subtheme 3e: priorities for fall risk reduction and fall prevention}

Participants believed that public awareness about invisible disabilities was important to improve accessibility and consequently reduce their risk of falls in the community. For instance, it is necessary to educate bus drivers to consider the needs of passengers with invisible disabilities. P1 shared his experience of falling after the transit driver accelerated too quickly: 'The driver just floored it. And I fell forward and I remember I hit my head.' With awareness of the potentially imperceptible needs of some passengers on public transport, situations like this one could be avoided.

In addition, participants believed PSWs should be educated on fall prevention as they work closely with individuals with SCI. The increased awareness and understanding by PSWs as well as by the public could greatly mitigate the risk of falling for participants with SCI. 


\section{DISCUSSION}

This is the first study to use a mixed-methods approach of quantitative surveys and qualitative photovoice methodology to investigate the perspectives of ambulators with SCI. This research provides insights into circumstances causing falls, the impacts of falls, and essential considerations for fall prevention initiatives. Over a 6-month period, nearly two-thirds of ambulators with SCI fell at least one time, and the majority of these participants fell multiple times. Given this high incidence rate, fall prevention interventions that can successfully reduce the frequency of falls while maintaining an individual's mobility and participation in meaningful activities are necessary.

Consistent with previous literature,${ }^{20}$ most falls occurred inside or just outside the home. One possible explanation for the fewer observed number of falls in the community is that participants may be more aware of fall risk factors in the community, allowing them to focus on avoiding these factors to proactively avoid falling. ${ }^{21}$ In contrast, within the home, participants may have limited 'risk awareness" ${ }^{2}$ of fall risk factors, as the home environment is very familiar. ${ }^{7}$ This was demonstrated in the photographs depicting risk factors, as many participants captured photographs of obvious community fall risk factors such as icy sidewalks. They described the importance of cognitive awareness to compensate for impaired bodily functions; however, this strategy requires a great deal of effort and concentration that may not always be possible. ${ }^{2}$

In the home environment, most falls occurred while participants were changing positions or walking, whereas in the community, falls tended to occur while participants were standing or walking. ${ }^{45}$ The influence of intrinsic factors such as poor balance and leg weakness were believed by participants to contribute to nearly twothirds of falls that occurred in the home. Conversely, poor balance, tripping and slipping were more heavily associated with falls in the community. These differences in risk factors suggest the necessity for distinct fall prevention strategies in the home compared with the community.

In the qualitative discussions, less obvious contributors including pain and fatigue were revealed as underlying causes of poor balance and leg weakness. More than half of people with SCI experience such high levels of fatigue that it interferes with their daily functioning. ${ }^{22}$ Individuals with SCI may experience excessive levels of fatigue after a task of $2-3$ hours, ${ }^{23}$ which may explain participants' falls during physical activities. Furthermore, since most falls experienced by ambulators with SCI occurred in the morning and afternoon, it is possible that falls during physically/complex demanding morning activities (showering, dressing, etc) were triggered by fatigue and/or morning stiffness. However, more research is needed to understand how fatigue can impact fall risk, and to explore some strategies to counteract fatigue. When investigating fall circumstances, clinicians should ask individuals with SCI whether excessive fatigue is a concern. If so, fatigue management strategies, such as recognising the symptoms, activity modifications, and rest breaks should be explored. ${ }^{24}$

Pain was previously identified as a risk factor for falls by wheelchair users. ${ }^{25}$ This study found that when pain was experienced in only one leg, it caused gait imbalance. Further, upper extremity pain interfered with participants' grasp of a gait or mobility aid. This is one possible reason for participants' inconsistent use of gait aids. Some participants consumed alcohol to reduce neuropathic pain, but since alcohol reduces balance and coordination this behaviour is problematic. ${ }^{26}$ This study highlights that in some cases, poor pain management can put a person at a higher risk of falling, and proper pain management should be explored in future fall prevention initiatives.

As expected, most falls occurred while participants were ambulating. Sixty-six per cent of people with SCI use at least one mobility aid, and many use multiple gait aids. $^{27}$ The participants who used multiple mobility aids had to judge which aid was suitable for a given situation, and their judgements were sometimes incorrect. The qualitative results also revealed that participants did not consistently use a gait aid in their homes. To reduce the risk of falling, it is important for clinicians to re-assess the suitability of gait aids and the consistency with which they are used by ambulators. Specific barriers to using a gait aid in the home should also be explored. For instance, walking with a gait aid (eg, a cane or walker) improves balance and mobility, however, long-term use of a gait aid can cause shoulder pain and fatigue. ${ }^{28} 29$ As well, walking devices can interfere with clearing obstacles which could lead to more falls. ${ }^{30}$ Another barrier to using a gait aid, such as a cane, walker, crutches is that it makes it difficult, even impossible, to perform daily activities that require carrying objects. ${ }^{31}$ These reasons may also contribute to participants' decisions not to use a gait aid consistently, particularly in their homes. Future research should investigate whether people who fell were using appropriate mobility aids and should provide ambulators with knowledge on how to recognise their changing mobility needs.

Another consideration is the gap in follow-up care of people with SCI-a person's mobility needs may change over time, but their mobility may not be adequately reassessed by a healthcare professional. ${ }^{32}$ Therefore, it is also possible that ambulators living with chronic SCI are using mobility aids that are not appropriate for their needs which could increase their risk of falling.

Similarly to wheelchair users with SCI, ${ }^{33}$ ambulators with SCI had varying levels of concern about falling. All participants believed that winter conditions increased their fall risk, and it was unsurprising that some ambulators with SCI limited their community participation in these conditions due to concerns that they might fall. ${ }^{26}$ In addition to the obvious fall risks in winter including slippery surfaces, cold weather triggered unpredictable body responses that increased an ambulator's risk of falling. Ambulators, clinicians and caregivers should exercise additional precautions to reduce the fall risk caused by these conditions. As some people may be fearful of going 
outdoors in harsh winter weather, if possible, telehealth consultations for medication appointments or accommodations at work ${ }^{33}$ should be explored.

A comprehensive approach to fall prevention will involve multiple layers of interventions at the individual level to target potentially modifiable contributors to falls. For instance, interventions/strategies can be explored to target 'poor balance'13 34 'weakness in legs', 35 'fatigue',24 and 'pain'. ${ }^{36}$ Fall risk factors, such as doing more than one thing, moving too quickly/rushing, not using mobility aids and being distracted, can be addressed using behavioural strategies. ${ }^{2}{ }^{9}$ Furthermore, environmental factors that contribute to falls, such as tripping over items in the environment or slipping can be addressed by environmental modifications. ${ }^{2}$ Unfortunately, even with interventions some falls are inevitable. Therefore, it is valuable for fall prevention interventions to teach individuals how to safely get up from a fall.

Many fall prevention strategies highlighted by ambulators with SCI were similar to the strategies used by wheelchair users with SCI. ${ }^{37}$ Also, other articulated strategies from this study are related to proper footwear, fatigue, and pain management. This study reinforces the notion that numerous factors can contribute to falls, and fall prevention must be a joined effort among physicians, therapists, ambulators, caregivers and employers. ${ }^{37}$ As some participants were sceptical of the utility of a fall prevention programme, a potential buy-in to fall prevention programmes should be further explored. This would allow interested ambulators to participate but would not compel anyone to receive training. In contrast to wheelchair users with $\mathrm{SCI},{ }^{37}$ many ambulators believe fall prevention efforts should be led by clinicians since they are the experts on fall prevention.

Participants spoke about an optimal level of caution, as both being overly cautious and under-cautious increased the risk of falling. For example, when people with SCI are over-cautious and have a fear of falling, it can adversely affect postural control and increase the risk of falls. ${ }^{9}$ As part of fall prevention, strategies to manage anxiety should be explored to reduce this risk. Achieving this optimal level of caution was a crucial and dynamic task, and participants appeared to increase their level of caution in situations that had obvious hazards (eg, in places they had previously fallen or in the community), and exhibit decreased cautiousness in situations perceived as 'low risk' for falls (eg, during transfers and mobility within their home).

Regardless, there are several limitations that must be considered when interpreting these findings. First, falls were tracked for a period of only 6 months. Participants commonly identified winter weather as a fall risk factor; however, since participants enrolled in this study at different times of the calendar year, the study did not quantitatively explore whether participants fell more during the winter compared with other seasons. Another limitation is that this study did not ask specifically where in the home or community participants had fallen, so conclusions could not be drawn about specific rooms or locations. In addition, a sequential exploratory design allowed us to integrate the quantitative and qualitative data; however, different sample sizes in phases 1 and 2 could impact the generalisability of the results/interpretations. Lastly, it is important to acknowledge that older individuals are over-represented in this study; the average age of the total sample in phase 1 is 61.6 years and most of the participants in phase 2 were aged mid to late 50. Considering that older and younger individuals engage in different activities, the results of this study may not be generalisable to a younger population. ${ }^{38}$

Overall, this mixed-methods approach allowed for the identification of factors related to fall circumstances in ambulators, and these new findings should be considered in future fall prevention initiatives. Creating effective and comprehensive fall prevention interventions for ambulators with SCI will not be an easy feat. Multiple factors must be considered, including the unique needs of the individual and the multiple factors that put them at an increased risk.

\section{CONCLUSION}

Although the home was a familiar environment, most falls occurred in the home. As such, fall prevention interventions/strategies for ambulators with SCI should consider the fall risk factors in a person's home and in all their day-to-day activities.

\section{Author affiliations}

${ }^{1}$ Rehabilitation Sciences Institute, University of Toronto Faculty of Medicine, Toronto, Ontario, Canada

${ }^{2}$ KITE, Toronto Rehabilitation Institute - University Health Network, Toronto, Ontario, Canada

${ }^{3}$ Neuroscience Program (interdepartmental), Department of Biology, Physiology, and Psychology, McGill University, Montreal, Québec, Canada

${ }^{4}$ Department of Physical Therapy, University of Alberta, Edmonton, Alberta, Canada ${ }^{5}$ Department of Physical Therapy, University of Toronto Faculty of Medicine, Toronto, Ontario, Canada

Acknowledgements We would like to thank Katherine Chan from the SCI Mobility Lab (TRI-UHN) for her assistance with transcription and the participants for dedicating their time to this study.

Contributors HS: conceptualisation, methodology, writing-original draft. ASR: writing-original draft, reviewing and editing. GB: methodology, Writingreviewing and editing. AK: methodology, writing-reviewing and editing. KEM: conceptualisation, methodology, supervision, writing-reviewing and editing.

Funding This work was supported by the Craig H. Neilsen Psychosocial Research Grant, grant number (440070) to KEM and student scholarships from Toronto Rehabilitation Institute and the Canadian Institutes of Health Research to HS.

Disclaimer The funders did not have a role in the study's design, execution, analyses or interpretation of the data.

Competing interests None declared.

Patient consent for publication Obtained.

Ethics approval The Research Ethics Boards of the UHN (14-8569-DE) and University of Toronto (34267) granted ethical approval for this study.

Provenance and peer review Not commissioned; externally peer reviewed.

Data availability statement Data are available on reasonable request. The datasets generated and/or analysed during the current study are not publicly 
available due to confidentiality of data, but are available from the corresponding author on reasonable request.

Open access This is an open access article distributed in accordance with the Creative Commons Attribution Non Commercial (CC BY-NC 4.0) license, which permits others to distribute, remix, adapt, build upon this work non-commercially, and license their derivative works on different terms, provided the original work is properly cited, appropriate credit is given, any changes made indicated, and the use is non-commercial. See: http://creativecommons.org/licenses/by-nc/4.0/.

\section{ORCID iD}

Hardeep Singh http://orcid.org/0000-0002-7429-5580

\section{REFERENCES}

1 Khan A, Pujol C, Laylor M, et al. Falls after spinal cord injury: a systematic review and meta-analysis of incidence proportion and contributing factors. Spinal Cord 2019;57:526-39.

2 Jørgensen V, Roaldsen KS. Negotiating identity and self-image: perceptions of falls in ambulatory individuals with spinal cord injury a qualitative study. Clin Rehabil 2017;31:544-54.

3 Nas K, Yazmalar L, Şah V, et al. Rehabilitation of spinal cord injuries. World J Orthop 2015;6:8-16.

4 Amatachaya S, Wannapakhe J, Arrayawichanon P, et al. Functional abilities, incidences of complications and falls of patients with spinal cord injury 6 months after discharge. Spinal Cord 2011;49:520-4.

5 Wannapakhe J, Arayawichanon P, Saengsuwan J, et al. Changes of functional ability in patients with spinal cord injury with and without falls during 6 months after discharge. Phys Ther 2014;94:675-81.

6 Brotherton SS, Krause JS, Nietert PJ. Falls in individuals with incomplete spinal cord injury. Spinal Cord 2007;45:37-40.

7 Musselman KE, Arnold C, Pujol C, et al. Falls, mobility, and physical activity after spinal cord injury: an exploratory study using photoelicitation interviewing. Spinal Cord Ser Cases 2018;4:39.

8 Jørgensen V, Butler Forslund E, Franzén E, et al. Factors associated with recurrent falls in individuals with traumatic spinal cord injury: a multicenter study. Arch Phys Med Rehabil 2016;97:1908-16.

9 Jørgensen V, Butler Forslund E, Opheim A, et al. Falls and fear of falling predict future falls and related injuries in ambulatory individuals with spinal cord injury: a longitudinal observational study. J Physiother 2017;63:108-13.

10 Child S, Goodwin V, Garside R, et al. Factors influencing the implementation of fall-prevention programmes: a systematic review and synthesis of qualitative studies. Implement Sci 2012;7:91.

11 O'Cathain A, Murphy E, Nicholl J. The quality of mixed methods studies in health services research. J Health Serv Res Policy 2008;13:92-8.

12 Wang CC. Photovoice: a participatory action research strategy applied to women's health. J Womens Health 1999;8:185-92.

13 Unger J, Chan K, Scovil CY, et al. Intensive balance training for adults with incomplete spinal cord injuries: protocol for an assessorblinded randomized clinical trial. Phys Ther 2019;99:420-7.

14 WHO. Falls, 2018. Available: https://www.who.int/news-room/factsheets/detail/falls [Accessed 14 Nov 2019].

15 Greenland S, Rothman KJ, 3rd ed. Measures of occurrence. In: Rothman KJ GS, Lash TL, eds. Modern epidemiology. Philadelphia, PA: Lippincott Williams \& Wilkins, 2008.

16 Braun V, Clarke V. Using thematic analysis in psychology. Qual Res Psychol 2006;3:77-101.
17 Shenton AK. Strategies for ensuring trustworthiness in qualitative research projects. EFI 2004;22:63-75.

18 Krefting L. Rigor in qualitative research: the assessment of trustworthiness. Am J Occup Ther 1991;45:214-22.

19 WHO. International classification of functioning, disability and health 2007. Available: https://apps.who.int/iris/bitstream/handle/10665/ 43737/9789241547321 eng.pdf;jsessionid=3463A191466EBB66 40BBBE7F25E6DABF? sequence=1 [Accessed 23 Jan 2020].

20 Phonthee S, Saengsuwan J, Siritaratiwat W, et al. Incidence and factors associated with falls in independent ambulatory individuals with spinal cord injury: a 6-month prospective study. Phys Ther 2013;93:1061-72.

21 Forslund EB. Falls in wheelchair users with spinal cord injury incidence, risks and concerns. Karolinska Institutet, 2017.

22 Fawkes-Kirby TM, Wheeler MA, Anton HA, et al. Clinical correlates of fatigue in spinal cord injury. Spinal Cord 2008;46:21-5.

23 Craig A, Tran Y, Wijesuriya N, et al. Fatigue and tiredness in people with spinal cord injury. J Psychosom Res 2012;73:205-10.

24 Hammell KW, Miller WC, Forwell SJ, et al. Managing fatigue following spinal cord injury: a qualitative exploration. Disabil Rehabil 2009;31:1437-45.

25 Nelson AL, Groer S, Palacios P, et al. Wheelchair-related falls in veterans with spinal cord injury residing in the community: a prospective cohort study. Arch Phys Med Rehabil 2010;91:1166-73.

26 Luo J. Effects of ethanol on the cerebellum: advances and prospects. Cerebellum 2015;14:383-5.

27 Saunders LL, Krause JS, DiPiro ND, et al. Ambulation and complications related to assistive devices after spinal cord injury. $J$ Spinal Cord Med 2013;36:652-9.

28 Jain NB, Higgins LD, Katz JN, et al. Association of shoulder pain with the use of mobility devices in persons with chronic spinal cord injury. $\mathrm{Pm} R$ 2010;2:896-900.

29 Ulkar B, Yavuzer G, Guner R, et al. Energy expenditure of the paraplegic gait: comparison between different walking AIDS and normal subjects. Int J Rehabil Res 2003;26:213-7.

30 Bateni H, Maki BE. Assistive devices for balance and mobility: benefits, demands, and adverse consequences. Arch Phys Med Rehabil 2005;86:134-45.

31 Resnik L, Allen S, Isenstadt D, et al. Perspectives on use of mobility AIDS in a diverse population of seniors: implications for intervention. Disabil Health J 2009;2:77-85.

32 Craven CB, Verrier M, Balioussis C, et al. Rehabilitation environmental scan atlas: capturing capacity in Canadian SCI rehabilitation. Vancouver, BC: Rick Hansen Institute, 2012

33 Singh H, Scovil CY, Yoshida K, et al. Capturing the psychosocial impacts of falls from the perspectives of wheelchair users with spinal cord injury through photo-elicitation. Disabil Rehabil 2020:1-10.

34 Tamburella F, Scivoletto G, Molinari M. Balance training improves static stability and gait in chronic incomplete spinal cord injury subjects: a pilot study. Eur J Phys Rehabil Med 2013;49:353-64.

35 Stone WJ, Stevens SL, Fuller DK, et al. Strength and step activity after eccentric resistance training in those with incomplete spinal cord injuries. Top Spinal Cord Inj Rehabil 2018;24:343-52.

36 Franz S, Schulz B, Wang $\mathrm{H}$, et al. Management of pain in individuals with spinal cord injury: guideline of the German-Speaking medical society for spinal cord injury. Ger Med Sci 2019;17:Doc05.

37 Singh H, Scovil CY, Yoshida K, et al. Factors that influence the risk of falling after spinal cord injury: a qualitative photo-elicitation study with individuals that use a wheelchair as their primary means of mobility. BMJ Open 2020;10:e034279.

38 Marcum CS. Age differences in daily social activities. Res Aging 2013;35:612-40. 